\title{
Hypopharyngeal Epithelioid Malignant Schwannoma Following Treatment of Hodgkin's Lymphoma
}

\author{
Sevgi Gözdaşoğlư ${ }^{1}$, Koray Ceyhan ${ }^{2}$, Evren Üstüner ${ }^{3}$, Serdar Akyar $^{4}$, Mutlu Doğan $^{5}$ \\ and Fikri İçii \\ ${ }^{1}$ Ankara University School of Medicine, Department of Pediatric Hematology-Oncology, Ankara, \\ Turkey \\ ${ }^{2}$ Ankara University School of Medicine, Department of Pathology, Ankara, Turkey \\ ${ }^{3}$ Ankara University School of Medicine, Department of Gastroenterology, Ankara, Turkey \\ ${ }^{4}$ Ankara University School of Medicine, Department of Radiology, Ankara, Turkey \\ ${ }^{5,6}$ Ankara University School of Medicine, Department of Medical Oncology, Ankara, Turkey
}

Correspondence should be addressed to: Sevgi Gözdaşoğlu; sgozdasoglu@hotmail.com

Received date: 10 May 2014; Accepted date: 24 July 2014; Published date: 25 June 2015

Academic Editor: Leila Antonângelo

Copyright (C) 2015. Sevgi Gözdaşoğlu, Koray Ceyhan, Evren Üstüner, Serdar Akyar, Mutlu Doğan and Fikri İçli. Distributed under Creative Commons CC-BY 4.0

\begin{abstract}
The objective of this study is to present a patient with hypopharyngeal epithelioid malignant schwannoma as a secondary malignant neoplasm following treatment with MOPP and radiotherapy. The patient, presently a 43 year-old man, was diagnosed at clinical stage III Hodgkin's lymphoma (HL) with mixed cellularity histopathology at the age of thirteen. He had received 40 Gy radiotherapy to the neck region and MOPP + maintenance MOPP (a total 10 cycles), while malignant schwannoma developed 30 years after the treatment. Although the patient further received four cycles of ifosfamide + adriamycin combination chemotherapy, he died with progression of his malignant schwannoma six months after diagnosis.
\end{abstract}

Keywords: Malignant schwannoma, treatment, Hodgkin's lymphoma, secondary neoplasia

\section{Introduction}

Developments in chemotherapy and radiotherapy have enabled most patients with HL to be cured. However, the longterm effects of anticancer treatment include an increased risk of second malignant neoplasms (SMNs). The incidence of SMNs has been extensively investigated in the treated cases of HL. A wide variety of SMNs have been reported, including leukemias, NHL and solid tumors, especially breast and thyroid cancers. Breast cancer was the most common solid tumor with an estimated actuarial incidence in women that approached 35\%

Cite this Article as: Sevgi Gözdaşoğlu, Koray Ceyhan, Evren Üstüner, Serdar Akyar, Mutlu Doğan and Fikri İçli (2015), "Hypopharyngeal Epithelioid Malignant Schwannoma Following Treatment of Hodgkin's Lymphoma ", International Journal of Case Reports in Medicine, Vol. 2015 (2015), Article ID 275309, DOI: 
by 40 years of age (Bhatia, et al., 1996). These SMNs occurred from 3 months to 21 years after the diagnosis of HL with leukemias, having a median latent period of 5.5 years and solid tumors 9.5 years from the time of diagnosis. The majority of SMNs developed in tissues exposed to radiotherapy (Meadows, et al., 1985). A few cases with malignant schwannoma after radiotherapy for HL were reported in the literature (Adamson, et al., 2004).

Malignant peripheral nerve sheath tumors (MPNSTs) are rare soft tissue neoplasms, including $5-10 \%$ of all soft tissue sarcomas, about one-fourth to one-half occurring with neurofibromatosis 1 (NF1) (Weiss and Goldblum, 2001; Layfield, 2002). The tumor originates usually from a preexisting nerve sheath tumor (i.e. neurofibroma) or a major nerve trunk. The epithelioid variant is an unusual form of MPNSTs with a poor prognosis and represents approximately $5 \%$ of MPNSTs
(Reis et al, 2002). The tumor is composed of predominantly or exclusively Schwann cells with a polygonal epithelioid appearance (Layfield, 2002).

An unusual case with hypopharyngeal epithelioid-malignant schwannoma (EMPNST) following treatment for $\mathrm{HL}$ is presented in the following.

\section{Case Report}

A 43-year old man presented with more than two-months-history of progressive dysphagia, dyspnea and weight loss. His medical history revealed that, he was diagnosed as clinical stage III HL with mixed cellular histopathology at the age of 13 and had received 40 Gy radiotherapy to the neck region and MOPP (mustargen + oncovin + procarbazine + prednisone) + maintenance MOPP (total 10 cycles). A swan-like neck was observed 12 years later after the treatment (Figure 1).

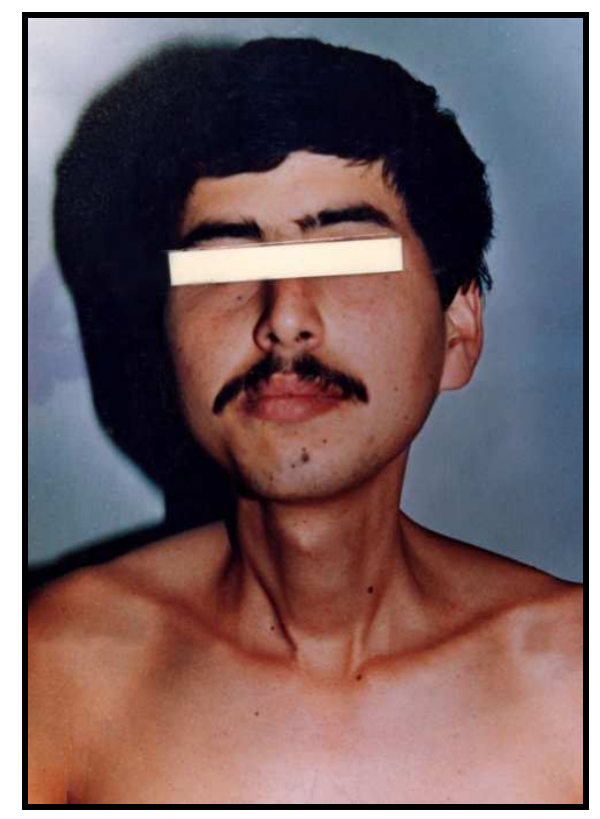

Figure 1: Appearance of swan-like neck

Cachexia and swan-like neck were noted on physical examination at his latest admission. The patient's esophagoscopic examination revealed a narrow segment between hypopharynx and cervical esophagus. No intraluminal mass was identified but a small mucosal erosion was reported as a mild chronic inflammation at biopsy. The computed tomography (CT) revealed a large infraglottic soft tissue mass, extending from the level of hypopharynx to the level of cervical 
esophagus. The tumor involved posterior hypopharyngeal wall and spreading to the adjacent trachea, thyroid gland, and cervical esophagus (Figure 2).

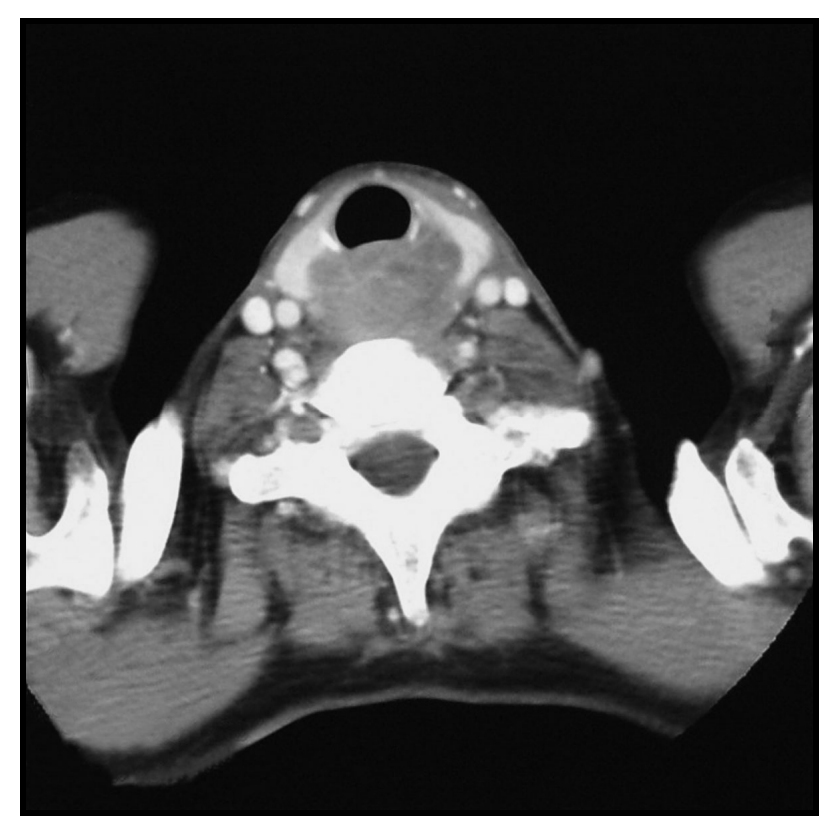

Figure 2: Axial CT image demonstrates a hypodense mass originating from the posterior hypopharyngeal wall infiltrating the esophagus, thyroid gland and trachea.

Thorax CT displayed enlarged paratracheal and subcarinal lymph-nodes and multiple small metastatic lung nodules. Cervical ultrasound imaging revealed a hypervascular and heterogeneous hypoechoic soft tissue mass invading the posterior thyroid gland. Tracheal rings and upper (cervical) esophageal invasion were also visualized, especially on the left side. Under ultrasound guidance, a percutaneous fine-needle aspiration biopsy was performed from the hypopharyngeal mass.

Cytopathologic examination smears revealed a hypercellular structure composed of isolated and dyscohesive polygonal and plasmacytoid epithelioid cells. The tumor cells had moderate nuclear pleomorphism, hyperchromatic and large central/paracentral nuclei with prominent nucleoli. Atypical mitotic figures and binucleated cells were commonly encountered. Relatively larger and pale cyanophilic cytoplasm with occasional microvacuoles were also observed (Figure 3) and cell block sections revealed irregular nodular epithelioid tumor cells within a fibromyxoid stroma (Figure 4). 


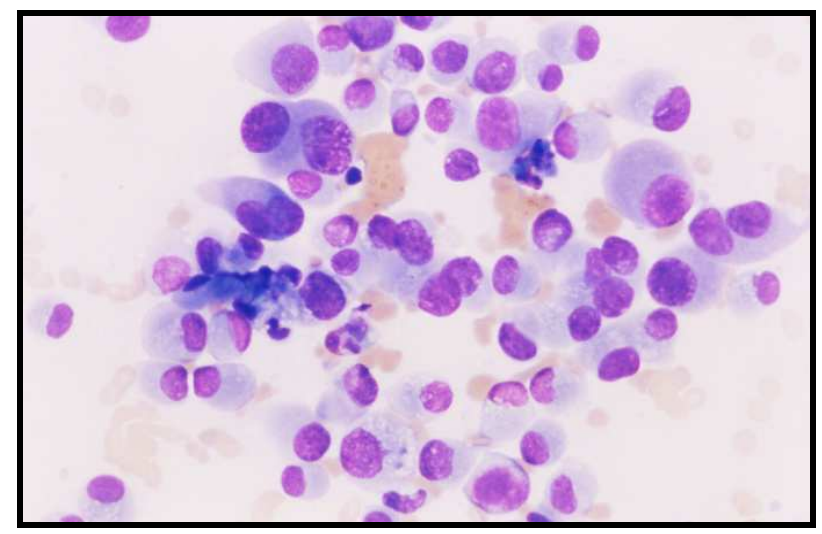

Figure 3: Binucleated tumor cells and cytoplasmic perinuclear small vacuoles (MGG stain, $X$ 200)

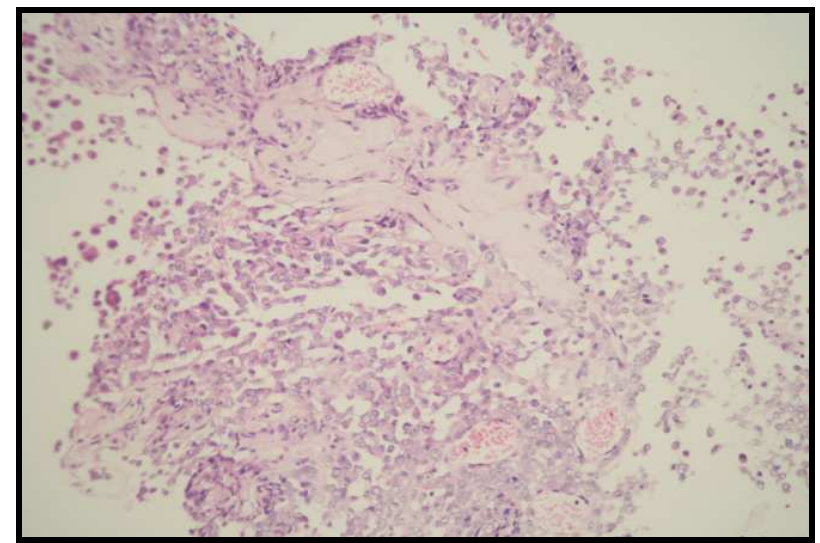

Figure 4: Cell block section of the tumor showing dyscohesive epithelioid tumor cells in a loose stroma (Hematoxylin-Eosin, $X$ 200)

Immunohistochemically, the tumor cells were strongly positive for vimentin, S-100 protein (Figure 5), CD57 (Leu-1), and GFAP (Figure 6).

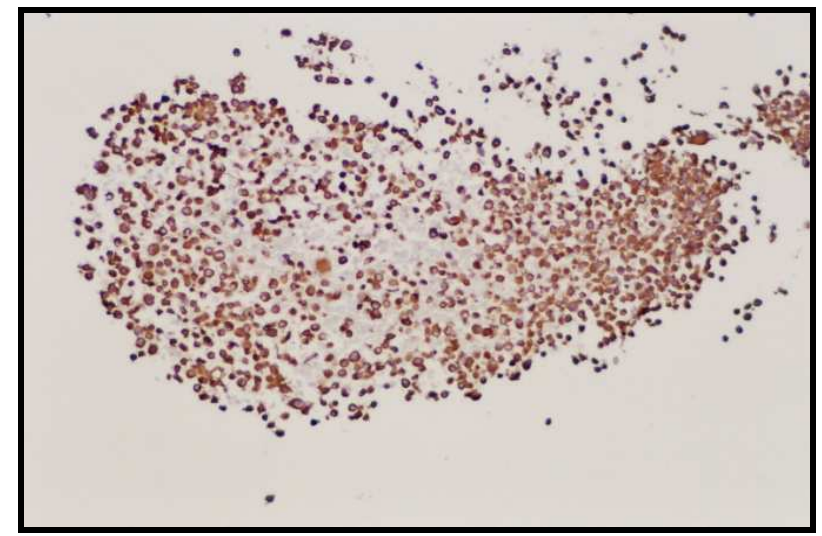

Figure 5: Diffuse nuclear and cytoplasmic S-100 protein positivity (DAB, X40) 


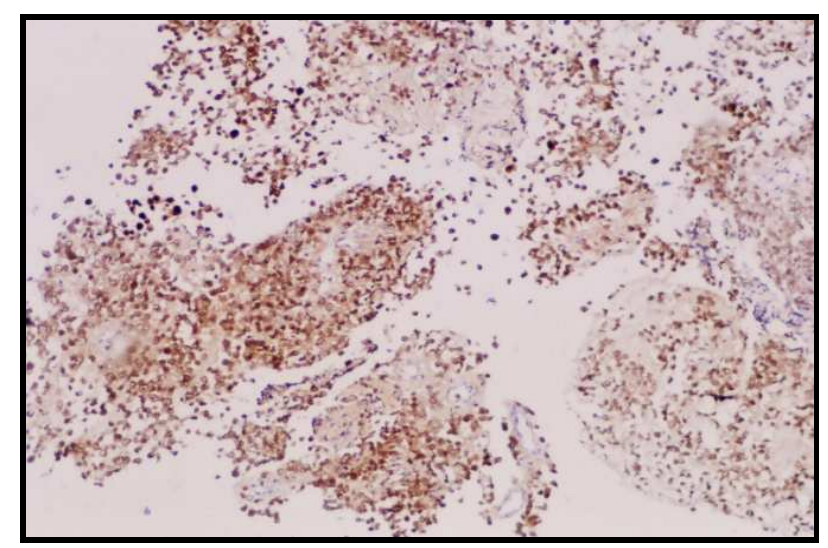

Figure 6: Diffuse cytoplasmic GFAP immunostaining (DAB, X100)

Pathologic diagnosis was epithelioid malignant schwannoma. The patient received a further four cycle ifosfamide + adriamycin combination chemotherapy, but remission could not be obtained. The patient died with progression of his disease 6 months after diagnosis.

\section{Discussion}

MPNSTs form a heterogenous group of neoplasms, originating from the different cells of the nerve sheath including Schwann cells, perineural cells, and fibroblasts (Layfield, 2002). The diagnosis of MPNSTs

requires at least one of the following criteria: (1) a tumor arising from a peripheral nerve usually a large nerve trunk, (2) a tumor originating from a preexisting benign nerve sheath tumor, (3) occurrence in a patient with neurofibromatosis or (4) definitive ultrastructural evidence of Schwann cell differentiation. In the absence of these features, it is possible to reach the correct diagnosis with the presence of classic morphologic features and characteristic immunohistochemistry findings (Weiss and Goldblum, 2001; Dodd et al., 1997). MPNST is a malignant mesenchymal neoplasm usually involving the extremities and the trunk. These tumors rarely involve the upper rodigestive system (Weiss and Goldblum, 2001; Layfield, 2002).

There is a limited number of studies on the cytological features of EMPNSTs. The Cytologic features of this tumor were first described by Molenaar et al. in 1989. Cytologically, these tumors usually consist of loosely cohesive clusters or dispersed large polygonal to plasmacytoid or epithelioid cells with prominent melanoma-like nucleoli. The tumor cells have generally moderate to large and finely granular cytoplasm with occasional microvacuolisation. Mitotic figures, prominent nuclear pleomorphism, and binucleation are other characteristic features (Dodd et al., 1997; Reis et al., 2002). Cyto/histomorphologic findings alone are not sufficient for EMPNST diagnosis. Obtaining a correct diagnosis is easier in patients with NF1 or when such a tumor arises from a major nerve or preexisting neurofibroma. In the absence of a suggestive clinical setting, on the basis of cyto/histomormorphologic features alone, the diagnosis of EMPNST is vague and immunocyto/histochemical and ultrastructural studies are essential to make a correct diagnosis and to exclude other possibilities. Strong immunostaining with S-100 protein, vimentin, GFAP or CD57 (Leu-7) are characteristic features for EMPNST (Dodd et al., 1997; Reis et al., 2002; Kwon et al., 2002).

A swan-like neck was seen in the patient 12 years later after the treatment and epithelioid malignant schwannoma developed in the irradiated area 30 years later after the radiotherapy. The patient could not achieve remission and died although four cycle ifosfamide + adriamycin combination chemotherapy were given. Adamson et al. (2004) reported 
two patients with MPNST of the spine after radiotherapy for Hodgkin's lymphoma and despite prompt surgical resection MPNSTs exhibited an aggressive behavior. The prognosis in the patients with MPNSTs is still poor.

On the other hand, in addition to chemoradiotherapy, genetic predisposing factors such as Li-Fraumeni syndrome, neurofibromatosis, genetic retinoblastoma have a potential for developing secondary neoplasms. It is also important to note that, genetic susceptibility may also play a role (Boice, 1996). Genomic analysis revealed alterations in cell cycle, repair, while detoxification and stress response pathways are suggested to be involved in the development of $\mathrm{HL}$ and in the occurrence of second neoplasias in these patients (Lorenzo et al., 2009).

M'Kacher et al. (2007) investigated the relationship between telomere shortening in peripheral blood lymphocytes, increased chromosome abnormalities, radiation sensitivity and secondary cancers. Prior to treatment, patients with HL showed ageindependent shorter telomeres, increased spontaneous chromosomal abnormalities and increased in-vitro radiation sensitivity. After treatment, telomere shortening was associated with cytogenetic profiles characterized by the persistence of complex chromosomal rearrangement and clonal aberrations. Patients with second cancers were characterized by markedly short telomeres, the presence of complex chromosome rearrangements and increased in vitro radiation sensitivity. An intimate relationship between pretreatment telomere shortening, chromosomal instability, radiation sensitivity and occurrence of second cancers was determined in the cases (M'Kacher et al., 2007).

The age at treatment has a major effect on risk of second malignancy after therapy for HL. A cohort of 5,519 patients with HL

treated during 1963-1993 was evaluated and followed-up for second malignancy. Three hundred twenty-two second malignancy occurred. Relative risks of cancers and of leukemia increased significantly with younger age at first treatment. However, absolute excess risks and cumulative risks of cancers and leukemia were greater at older ages. Although absolute excess risks are greater for older patients, relative risks of several important malignancies are much greater for patients who were treated when young (Swerdlow et al., 2000).

\section{Conclusion}

The clinicians should be aware of and alert for SMNs, especially in the patients treated with radiotherapy. Monitoring for the detection of SMNs in the survivors of childhood cancer necessitates a good collaboration between pediatric and adult oncology departments.

\section{References}

1. Adamson, D. C., Cummings, T. J. and Friedman, A.H. (2004) "Malignant peripheral nerve sheath tumor of the spine after radiation therapy for Hodgkin's lymphoma," Clinical Neuropathology, 23 (5) 245-255.

2. Bhatia, S., Robison, L. L., Oberlin, O., et al. (1996) "Breast cancer and other second neoplasms after Childhood Hodgkin's disease," The New England Journal of Medicine, 334 (12) 745-751.

3. Boice Jr., J.D. (1996) Cancer following irradition in childhood and adolescence, In: Green, D. M. and D'Angio, G. J., editors. "Mutagenic and carcinogenic effects of treatment for childhood cancer," Medical and Pediatric Oncology, (suppl 1) 29-35.

4. Dodd, L. G., Scully, S. and Layfield, L. J. (1997) "Fine-needle aspiration of epithelioid malignant peripheral nerve sheath tumor (epithelioid malignant schwannoma)," Diagnostic Cytopathology, 17 (3) 200-204.

5. Kwon, M. S., Lee, S. S. and Ahn, G. H. (2002) "Schwannomas of the gastrointestinal tract: clinicopathological features of 12 cases including a case of esophageal tumor compared with those of gastrointestinal stromal tumors and 
leiomyomas of the gastrointestinal tract," Pathology Research and Practice, 198 (9) 605-613.

6. Layfield, L. J. (2002) Cytopathology of Bone and Soft Tissue Tumors, Oxford University Press, New York, USA.

7. Lorenzo, Y., Provencio, M., Lombardia, L., et al. (2009) "Differential genetic and functional markers of second neoplasias in Hodgkin's disease patients," Clinical Cancer Research, 15 (15) 4823-4828.

8. M'kacher, R., Bennaceur-Griscelli, A., Girinsky, T., et al. (2007) "Telomere shortening and associated chromosomal instability in peripheral lymphocytes of patients with Hodgkin's lymphoma prior to any treatment are predictive of second cancers," International Journal of Radiation Oncology Biology Physics, 68 (2) 465-471.

9. Meadows, A. T., Baum, E., FossatiBellani, F., et al. (1985) "Second malignant neoplasms in children:An update from the late effects study group," Journal of Clinical Oncology, 3 (4) 532-538.
10. Molenaar, W. M., Ladde, B. E. Scraffordt Kroops, H., et al. (1989) "Two epithelioid malignant schwannomas in a patient with neurofibromatosis. Cytology, histology and DNA-flow-cytometry," Pathology Research and Practice, 184 (5) 529-534.

11. Reis Filho, J. S., Pope, L. Z., Balderrama, C. M., et al. (2002) "Epithelioid malignant peripheral nerve sheath tumour: case report and review of the previously published cases," Cytopathology, 13 (1) 5463.

12. Swerdlow, A. J., Barber, J. A., Hudson, G. V., et al. (2000) "Risk of second malignancy after Hodgkin's disease in a collaborative British cohort: the relation to age at treatment," Journal of Clinical Oncology, 18 (3) 498-509.

13. Weiss, S. W. and Goldblum, J. R. (2001), Malignant tumors of the peripheral nerves, In: Weiss, S. W. and Goldblum, J. R., editors. Soft Tissue Tumors. $4^{\text {th }}$ ed. St Louis, CV Mosby, 1209-1263. 